\title{
Social contagion in a world with asymmetric influence
}

\author{
G. S. Halvorsen $\odot,{ }^{*}$ B. N. Pedersen, and K. Sneppen \\ Copenhagen University, Niels Bohr Institute, Blegdamsvej 17, 2100 Copenhagen, Denmark
}

(Received 8 July 2020; accepted 12 November 2020; published 3 February 2021)

\begin{abstract}
Social media has blurred the distinction between news outlets and social networks by giving everyone access to mass communication. We simulate how influencers compete for attention on a social network by spreading information. The network structure occupies an ordered metastable state where one influencer maintains dominance for a sustained period or a fragmented state that divides attention between influencers. Numerical simulations are performed to map the domain of the ordered regime on various network topologies. Mutual coexistence between a few dominating influencers occurs on a scale-free social network. Our findings suggest the perception of fake news as a pervasive problem is endemic to a society where everyone can become a news outlet.
\end{abstract}

DOI: 10.1103/PhysRevE.103.022303

Social contagion processes have been simulated with local and reciprocal interactions between neighbors in a connected graph [1-5]. González-Avella et al. modeled the effect of mass media on the dissemination of culture [6-8]. Social media has disaggregated media influence by facilitating information sharing. Ordinary people can now communicate with unlimited subscribers, something previously reserved for news corporations. Members of a global social network can have as many subscribers as local newspapers or even large news corporations. We argue that users in a social network are now effectively news outlets, varying in size and impact. The capacity to interact with many is a defining property of the information age. The nature of this interaction is not reciprocal but directed; a collection of subscribers can receive information from a news outlet or an influencer, but there is no mutual exchange of information. Below, we simulate the emerging dynamics of a social network where any node can become an influencer and collect followers by sharing information. Our model integrates local reciprocal interactions between neighbors in a social network and global directed interactions between influencers and subscribers.

Before giving a formal description of the model, it is useful to describe its core mechanics in words. Consider a static undirected social network where nodes can be either followers or influencers. The topology of the social network has to be specified. A lattice is used to model the social network during the preliminary analysis, but other network topologies are considered toward the end. The basic idea is that influencers can build up a network of followers by sharing newsworthy information. Each follower is allowed to maintain only one directed link to an influencer at any point in time because

\footnotetext{
*gustavhalvorsen@nbi.ku.dk
}

Published by the American Physical Society under the terms of the Creative Commons Attribution 4.0 International license. Further distribution of this work must maintain attribution to the author(s) and the published article's title, journal citation, and DOI. attention is a finite resource [9]. Unlike the static links in the social network, these directed links can change ownership when a follower node decides to follow another influencer. Each node is assigned a news value $\tau$, gauging its knowledge of current affairs. The network is updated in discrete steps. For each update, a random node $a$ communicates with a random neighbor $b$ in its social network. If $b$ has a lower news value than $a$, then $b$ unfollows its own influencer to follow $a$ 's influencer. If the neighbor $b$ being attacked is an influencer, $b$ will instead copy $a$ 's news value onto itself and its own followers.

Each node has a probability $p_{i}$ of discovering a news event with every update. Galtung and Ruge [11] proposed a set of criteria known collectively as news values that influence the selection of published news. We quantify newsworthiness by assigning a news value of $\tau=t$ to a node that discovers a news event. Followers that discover news events are promoted to influencers. Influencers also update the news value of their subscribers to $t$ upon discovering a news event. Recent news events are thus assigned a higher news value to ensure that new information takes precedence. The emergence and decay of novelty have been the subject of empirical and theoretical modeling studies [12-15].

Figure 1 shows the possible interactions on a simple lattice. Figure 1(a) shows the discovery of a news event followed by the capture of a node, and Fig. 1(b) shows how an influencer can immunize its followers by appropriating information.

\section{MODEL}

We consider $N$ nodes connected by fixed links representing person to person communication in a static social network. A node can be either a follower $f$ or an influencer $S$. Each node has a current information state $\tau$ that is equal to the time of origin of the last news update it has received. Furthermore, each node is assigned an index, $i, j, \ldots, n$, that shows which influencer it belongs to. This is just a way to keep track of different subcultures. For example, if an influencer $a_{i} \in S$ has index $i$, then all of its followers will inherit this index. The 

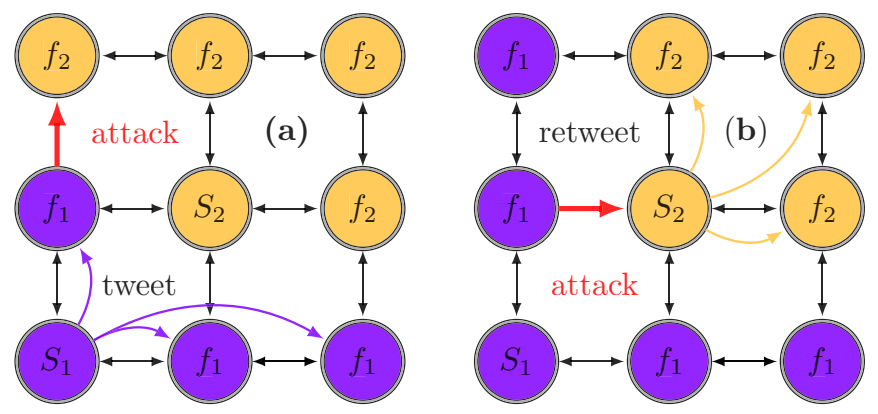

FIG. 1. Diagram of the model on a quadratic lattice. Each node represents a user on an undirected social network. Color and subscript indicate the follower's adherence to a news outlet. Panel (a) shows the discovery of a news event followed by the capture of a node. Panel (b) shows how an influencer $\left(S_{2}\right)$ can immunize its followers by copying information.

probability that a node generates a news event is given by

$$
p_{i}= \begin{cases}p_{0}\left(1+\eta k_{i}\right), & \text { influencer, } \\ p_{0}, & \text { otherwise }\end{cases}
$$

where $k_{i}$ is the number of followers the influencer with index $i$ presides over. Note that we define $p_{0}$ to be sufficiently small such that $p_{i}$ never exceeds unity, i.e., $p_{i}=p_{0}\left(1+\eta k_{i}\right) \leqslant 1$. Here $\eta$ is a positive feedback parameter between news generation and the number of subscribers [16]. Keep in mind that an influencer itself is a node and can therefore have no more than $N-1$ followers. Therefore, if $p_{0}=1 / N$ and $\eta=1.0$ then $p_{i}=\frac{1+(N-1)}{N}=1$. To avoid $p_{i}$ exceeding 1 , we impose the following bounds, $\eta \leqslant 1$ and $p_{0} \leqslant \frac{1}{N}$, on the model's parameters.

Time $t$ is advanced by one increment $t \rightarrow t+1$ for each $N$ updates performed on the system. To perform an update, do the following.

(i) Select a random node $a$. The chosen node discovers a news event with probability $p_{i}$ if it is an influencer or $p_{0}$ otherwise. If a follower discovers a news event, it is promoted to an influencer, i.e., its state is changed from $f$ to $S$, and it gains the news value $\tau=t$. If an influencer discovers a news event, it updates the news value of itself and its followers to $\tau=t$.

(ii) Proceed by selecting a random neighbor $b$ to the node $a$. Let $\tau(a)$ and $\tau(b)$ be the news values of $a$ and $b$, respectively. If $b$ is not an influencer and $\tau(a)>\tau(b)$, then $b$ adopts the news value $\tau(a)$ and begins to follow the same influencer as $a$. If $b$ is an influencer and $\tau(a)>\tau(b)$, then $b$ sets the news value of itself and its followers to $\tau(a)$.

The system is initialized at time $t=0$ with all nodes occupying the follower state. These unclaimed followers have not been assigned a news value $\tau$ and can be captured as soon as a node discovers the first news event. News events that occur later on are assigned a higher news value because $t$ increases as we perform more updates on the system. This mechanism is necessary to ensure that recent news events overwrite outdated ones. To prevent the system from freezing up with influencer nodes impervious to capture, we also demote influencers without any subscribers to the follower state. This is done whenever a full iteration of $N$ updates has been performed. These demoted influencers are not following anything but can be absorbed by another subculture. As a result of this removal process, the number of influencers will eventually saturate around some steady-state value that depends on $p_{0}$ and $N$ and on whether the system is ordered or fragmented.

\section{RESULTS}

Figure 2 show a simulation on a small system with a lattice topology and parameters $p_{0}=2.5 \times 10^{-4}$ and $\eta=0.0$ over 1100 time step iterations. Figure 2(a) shows the size of each subculture. The system occupies a perpetually fragmented state in the absence of feedback. Heterogeneity in the distribution of newsworthy information drives the conflict between influencers. To quote William Gibson, "The future is already here-it's just not evenly distributed." An influencer must discover new information to stay relevant and hold on to its followers. A newspaper that printed the same stories every day would presumably also lose its subscribers. Figures 2(b) and 2(c) show snapshots of the simulation. The system has self-organized into a configuration where influencers (white
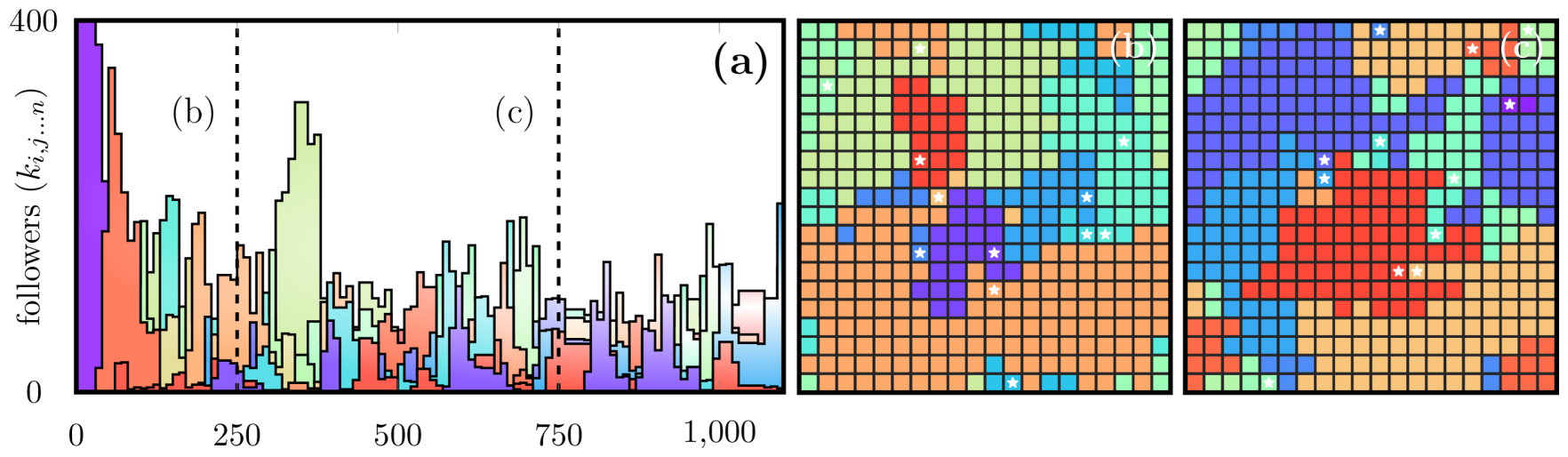

FIG. 2. Simulation on a quadratic lattice. Panel (a) shows the size of each subculture over 1100 time steps. Panels (b) and (c) each show a snapshot of the simulation at the labeled points in time. Each color defines a subculture, and the white stars represent influencers. The system occupies a perpetually fragmented state where no subculture dominates for an extended period. The simulation was carried out with periodic boundary conditions and parameters: $N=20 \times 20, p_{0}=2.5 \times 10^{-4}$, and $\eta=0.0$. Note that stars are only updated each time a full iteration of 400 updates has been performed. A full animation of the simulation is included as Supplemental Material [10]. 


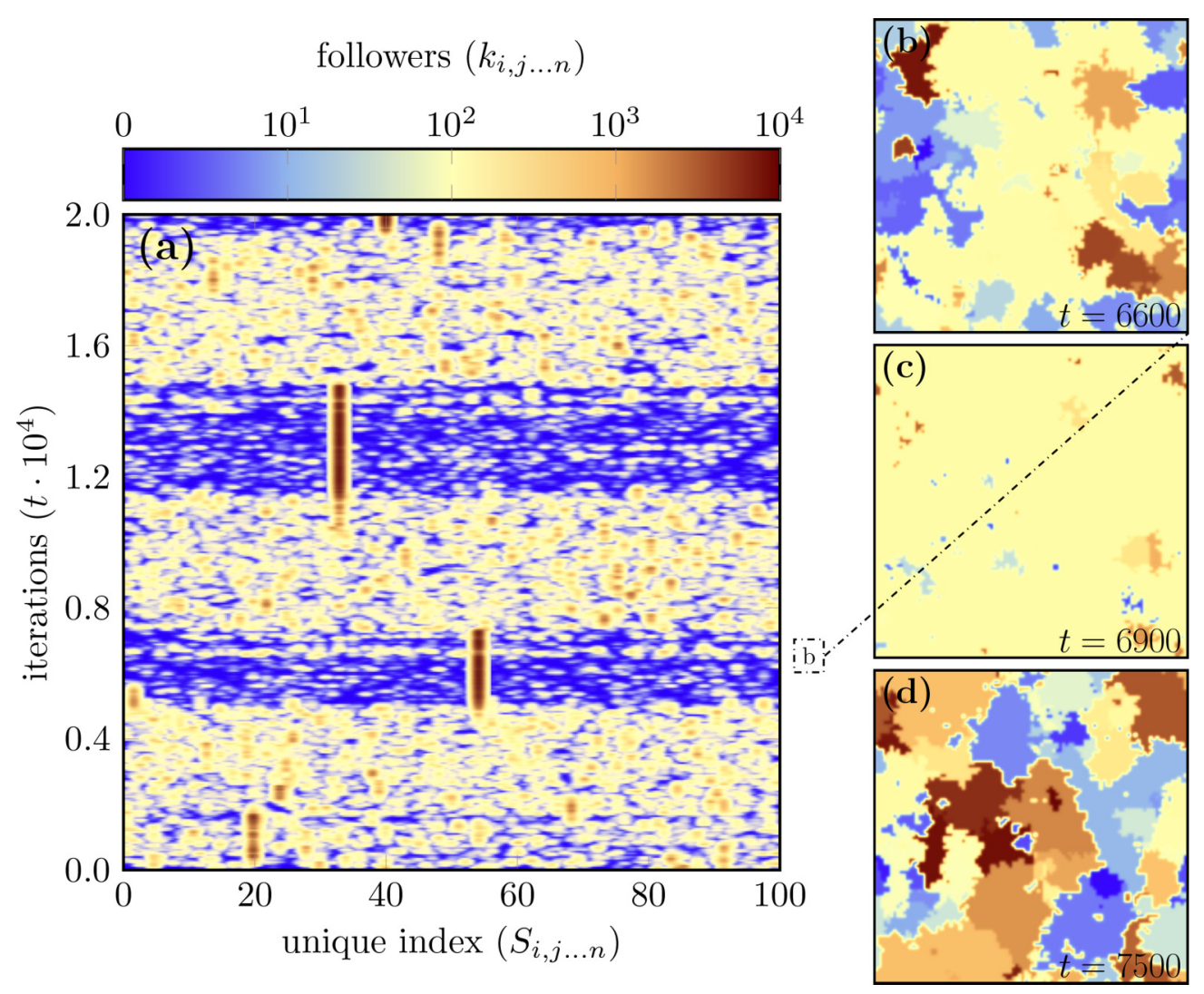

FIG. 3. Simulation with positive feedback on a quadratic lattice. Panel (a) shows the size of each subculture. Each vertical line represents the time evolution of a subculture. The solid lines are metastable states where the vast majority of followers belong to the same influencer. The diffuse blankets are fragmented states encompassing many subcultures of varying sizes. Panel (a) shows the first ordered metastable state emerges around $t=5 \times 10^{3}$. Panels (b)-(d) show static snapshots of the simulation at different points in time. Panel (b) shows the monoculture on the verge of collapse as many emerging subcultures capture followers from the dominating influencer. The largest subculture makes a speedy recovery in panel (c) before collapsing in panel (d) into a fragmented state. Simulation was carried out with periodic boundary conditions and the following set of parameters: $N=100 \times 100, \eta=0.30$, and $p_{0}=4.0 \times 10^{-5}$. The color scheme in panel (a) displays a subculture's size, but in the remaining panels, it displays affiliation with identical color nodes belonging to the same subculture.

stars) are located on the boundary between subcultures. An influencer must be attacked directly before it can copy information from its attacker. Following the appropriation of a news event, the two influencers and their followers will have the same news value. This immediately suspends the conflict until another news or appropriation event induces a discrepancy in news value between them.

Figure 3 shows a simulation on a larger system with parameters $p_{0}=4.0 \times 10^{-5}$ and $\eta=0.30$ over 20000 time-step iterations. Figure 3(a) shows the size of each subculture over the simulation. An influencer can now increase its probability of discovering a news event by capturing more followers. This positive feedback loop induces metastable states where one influencer maintains dominance for a sustained period.

Figures 3(b)-3(d) show snapshots of the simulation. Figure 3(a) shows that the first ordered metastable state emerges around $t=5 \times 10^{3}$. Figure $3(\mathrm{~b})$ reveals that the ordered state is about to collapse as many emerging subcultures capture followers from the dominating influencer. The monoculture makes a swift recovery in Figure 3(c) before it finally collapses into a fragmented state in Fig. 3(d). The transitions between the ordered and the fragmented regime are shortlived compared to the particular state's lifetime. These shifts occur over a few hundred iterations for the chosen parameters, whereas the fragmented or ordered states tend to persist for thousands of iterations. Increasing $\eta$ above a critical threshold eliminates the fragmented regime, but the system can still jump between different metastable states. The lifetime of a metastable state also increases with $\eta$. We also performed simulations on a one-dimensional system where no value of feedback was sufficient to induce ordered metastable states. This is consistent with van Hove's nonexistence theorem [17].

We use a linear feedback loop that increases $p_{i}$ with the number of subscribers $k_{i}$, but other functional dependencies were also considered. Sublinear growth is usually insufficient to support an ordered regime unless $\eta$ is increased to compensate. Let us assume that $N=10^{4}, p_{0}=1 / N$, and $\eta=1.0$. So if $p_{i}$, as an example, increases with the square root of $k_{i}$, an influencer with $N-1$ followers will have a $p_{i}$ value of 0.1 instead of 1.0 obtained with the linear model. However, faster than linear growth does not necessarily support an ordered regime either. If $p_{i}$ increases with the square of $k_{i}$, each influencer with 100 or more followers obtains a $p_{i}$ value greater than 1.0. This caps the advantage of followers above a critical threshold, as there is no benefit to a $p_{i}$ value greater than 1. 

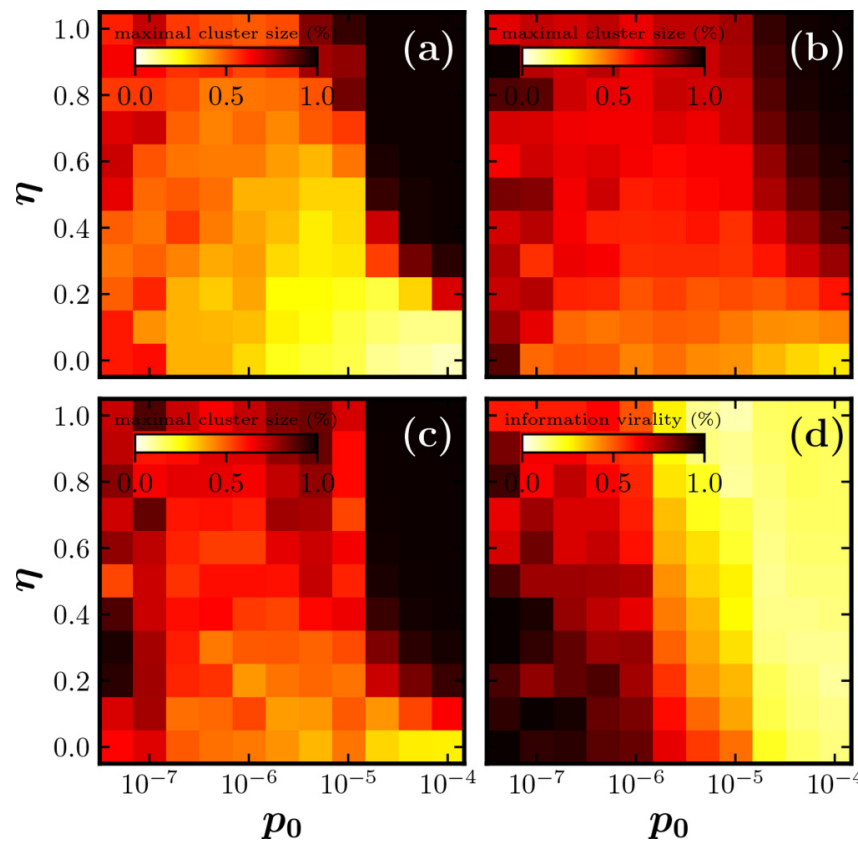

FIG. 4. Semilogarithmic phase diagrams. Panels (a)-(c) shows the time-averaged fraction of nodes belonging to the largest subculture for different network substrates. The followers in the largest subculture are counted for each time step and averaged over the full simulation. The subculture being sampled is not necessarily the same over the entire simulation. Panel (a) shows the quadratic lattice. Panel (b) shows a complete graph with interactions between random nodes. Panel (c) shows a small-world graph constructed by a Watts-Strogatz process: Each node is initially assigned four neighbors, and the links are randomly rewired between nodes with a probability of $\beta=0.1$. Dark regions represent the ordered domain in each diagram, and the system becomes increasingly disordered with brightness. The quadratic lattice becomes fully disordered in the lower-right corner, whereas the other network substrates become fragmented. The ordered domain is smaller for random mixing than for local interactions. Panel (d) shows the percentage of viral news events that spread to half or more of the nodes on a quadratic lattice. Each simulation was carried out with $N=10^{4}$ nodes and averaged over 10000 time steps.

Figure 4 shows a collection of phase diagrams. Figures 4(a)-4(c) show the time-averaged size of the largest subculture for different network topologies. Figure 4(a) shows the familiar quadratic lattice with local interactions. Figure 4(b) shows a complete graph with interactions between random nodes, i.e., mean-field behavior. Figure 4(c) shows a small-world graph constructed by a Watts-Strogatz model where each node is initially assigned four neighbors. The links are then randomly rewired with a probability of $\beta=0.1$. Social networks have small-world properties [18]. However, findings by Newman and Park suggest that real-world social networks differ from other networks in important ways [19]. All the network substates have an ordered regime for high values of $\eta$ and $p_{0}$. Both variables contribute because $p_{i}$ is increased by an increment of $\eta p_{0}$ for each follower gained. Dark regions represent the ordered domain in Figs. 4(a) to 4(c). This region is larger on the lattice than on the complete graph. Emerging subcultures can grow exponentially on a complete graph, whereas local interactions permit only polynomial growth. Simulations on the lattice thus give the influencer of the largest subculture more time to discover a news event before losing a critical number of followers. News events become less frequent as we decrease $p_{0}$, which permits an emerging influencer to capture a large number of followers before the next news event occurs, explaining why the maximal cluster size increases for very low $p_{0}$. This effect is also more pronounced on the complete and small-world graph than on the lattice, where news events take longer to spread.

Figure 4(d) shows the fraction of viral news events that spread to at least half of the system on a quadratic lattice. A news event has a good chance of capturing the attention of a broad audience when $p_{0}$ is small. However, competition intensifies as $p_{0}$ is increased, and more recent news events often overwrite news events before they can spread to a large fraction of the system. For large $p_{0}$ only a small fraction of events go viral [20]. So if almost nothing happens, the few exciting events that occur are very likely to receive widespread attention. But in a world exposed to a vast flux of news events, only a tiny fraction of these events get viral coverage. Figures 4(a) and 4(d) show that information diversity is suppressed in the ordered regime, as only news events propagated by the dominating influencer receive a broad audience.

We also performed a preliminary analysis on a scale-free network [21]. The Barabási-Albert model was used to grow a scale-free network of $N=10000$ nodes [21]. Each node is initially assigned four links that are preferentially attached to existing nodes with high degrees. As a result, a few nodes end up with disproportionately many links. Our analysis shows that mutual coexistence between a handful of dominating influencers emerges over time in the absence of positive feedback. The appropriation of news events was primarily a defensive mechanism on the lattice network. However, this mechanism can also be used offensively on a scale-free network. A node with many static links can easily pick up news events and use the information to attack other nodes together with its followers. Attacking these influencers' followers is difficult because their many links ensure that their news value is almost always up to date. We deemed a comprehensive analysis of the scale-free network to be prohibitively timeconsuming. Simulations must be averaged over a long time because the nodes with many links have only a small probability of being promoted to influencers.

\section{DISCUSSION}

Our work concerns competition between news outlets in an information supply economy with finite attention. We have shown that a benefit to size can lead to a metastable state where one news outlet dominates. Shifts between stable states are often interspersed with periods of fragmentation. The collapse of the ordered state occurs when many new subcultures emerge and capture followers from the dominant influencer, decreasing its probability of discovering news. If it fails to update its news value, it will also continue to bleed subscribers, and the system collapses into a fragmented state. This represents a transformation from a centralized form of media influence to a disaggregated collection of independent 
news outlets. We argue that ubiquitous information sharing has brought about a similar change in our society. Three news networks used to dominate mass media in the United States [22]. Easy access to communication has driven down the cost of becoming a news outlet or influencer, dramatically increasing $p_{0}$. The monetary benefit of being a news outlet has also diminished, meaning that $\eta$ assumes lower values in a world where mass communication is available to everyone. For example, the rise of digital media explains the decline of local newspapers [23].

There is growing concern that the internet has brought about an epidemic of fake news by lowering the cost of entry to new competitors, many of whom reject journalistic norms [24]. Vosoughi et al. [25] showed that fake news stories spread more than the truth. Fake news outlets can fabricate stories of greater newsworthiness, so it should not be surprising that false news spreads more than the truth. However, some studies dispute the prevalence of fake news. Findings by Allen et al. [26] suggests that fake news only makes up a small percentage of Americans' daily media diets. True or false, the perception of fake news as being a huge issue is widespread. The advent of social media can explain this phenomenon. Residents of a polarized society may think that fake news is pervasive because information diversity is high. The inhabitants of a monoculture may be less inclined to share the perception of fake news as a problem-even if the dominating influence is passing on false information. Mass media in autocratic societies fits this description particularly well; a single news outlet effectively controls the flow of information, and the information is, we assume, overwhelmingly false. If so, it is information diversity that precipitates the perception of fake news.

The information content of news events was not modeled explicitly in this work, nor did we differentiate between real and fake news outlets or influencers. An extension of the model could assign a higher $p$ value to fake news outlets. Introducing a measure of credibility would be necessary to penalize outlets that disseminate fake news. Respectable news outlets would then find themselves in a delicate situation when deciding whether or not to pass on information from another source. The risk of passing on false information entails a loss of reputation. However, journalists often face this dilemma because the race to report on a breaking news event first can drive even respectable news outlets to publish information before adequately vetting it.

To summarize, we model a network that exhibits transitions between a dominating media influence and a decentralized collection of independent news outlets. Our findings suggest the perception of fake news as a widespread problem is endemic to a society where everyone can become a media outlet.

\section{ACKNOWLEDGMENTS}

This project has received funding from the European Research Council (ERC) under the European Union's Horizon 2020 Research and Innovation Program, Grant Agreement No. 740704.
[1] P. Clifford and A. Sudbury, Biometrika 60, 581 (1973).

[2] K. Sznajd-Weron and J. Sznajd, Int. J. Mod. Phys. C 11, 1157 (2000).

[3] R. Hegselmann, U. Krause et al., J. Artif. Soc. Soc. Stimul. 5 (2002).

[4] R. A. Holley and T. M. Liggett, The annals of probability 3, 643 (1975).

[5] R. Axelrod, J. Conflict Resolut. 41, 203 (1997).

[6] J. C. González-Avella, M. G. Cosenza, and K. Tucci, Phys. Rev. E 72, 065102(R) (2005).

[7] J. C. González-Avella, V. M. Eguíluz, M. G. Cosenza, K. Klemm, J. L. Herrera, and M. San Miguel, Phys. Rev. E 73, 046119 (2006).

[8] K. I. Mazzitello, J. Candia, and V. Dossetti, Int. J. Mod. Phys. C 18, 1475 (2007).

[9] L. Weng, A. Flammini, A. Vespignani, and F. Menczer, Sci. Rep. 2, 335 (2012).

[10] See Supplemental Material at http://link.aps.org/supplemental/ 10.1103/PhysRevE.103.022303 for full animation of Fig. 2 displayed at 8 frames per second.

[11] J. Galtung and M. H. Ruge, J. Peace Res. 2, 64 (1965).

[12] F. Wu and B. A. Huberman, Proc. Natl. Acad. Sci. USA 104, 17599 (2007).

[13] L. Lizana, M. Rosvall, and K. Sneppen, Phys. Rev. Lett. 104, 040603 (2010).
[14] L. Lizana, N. Mitarai, K. Sneppen, and H. Nakanishi, Phys. Rev. E 83, 066116 (2011).

[15] S. Bornholdt, M. H. Jensen, and K. Sneppen, Phys. Rev. Lett. 106, 058701 (2011).

[16] W. B. Arthur, Sci. Am. 262, 92 (1990).

[17] L. Van Hove, Physica (Amsterdam) 16, 137 (1950).

[18] D. J. Watts and S. H. Strogatz, Nature (London) 393, 440 (1998).

[19] M. E. J. Newman and J. Park, Phys. Rev. E 68, 036122 (2003).

[20] S. Asur, B. A. Huberman, G. Szabo, and C. Wang, in Proceedings of the Fifth International AAAI Conference on Weblogs and Social Media (AAAI, Palo Alto, California, 2011).

[21] R. Albert and A.-L. Barabási, Rev. Mod. Phys. 74, 47 (2002).

[22] D. B. Hindman and K. Wiegand, J. Broadcast. Electron. Media 52, 119 (2008).

[23] R. K. Nielsen, Local Journalism: The Decline of Newspapers and the Rise of Digital Media (Bloomsbury, London, 2015).

[24] D. M. Lazer, M. A. Baum, Y. Benkler, A. J. Berinsky, K. M. Greenhill, F. Menczer, M. J. Metzger, B. Nyhan, G. Pennycook, D. Rothschild et al., Science 359, 1094 (2018).

[25] S. Vosoughi, D. Roy, and S. Aral, Science 359, 1146 (2018).

[26] J. Allen, B. Howland, M. Mobius, D. Rothschild, and D. J. Watts, Sci. Adv. 6, eaay3539 (2020). 